TITLE:

\title{
Interference of spherical wave of thermal radiation emitted by a film system
}

$\operatorname{AUTHOR}(S):$

WAKABAYASHI, Hidenobu; MAKINO, Toshiro

\section{CITATION:}

WAKABAYASHI, Hidenobu ... [et al]. Interference of spherical wave of thermal radiation emitted by a film system. International Journal of Heat and Mass Transfer 2008, 51(11-12): 2762-2771

ISSUE DATE:

2008-06

URL:

http://hdl.handle.net/2433/91246

\section{RIGHT:}

Copyright @ 2007 Elsevier; この論文は出版社版でありません。引用の 際には出版社版をご確認ご利用ください。; This is not the published version. Please cite only the published version. 


\section{Interference of Spherical Wave of Thermal Radiation Emitted by a Film System}

Hidenobu Wakabayashi and Toshiro Makino

Department of Mechanical Engineering and Science,

Kyoto University, Kyoto 606-8501, Japan.

Corresponding author: Hidenobu Wakabayashi

$$
\begin{aligned}
& \text { Telephone }+81-(0) 75-753-5262 \\
& \text { Facsimile }+81-(0) 75-771-7286
\end{aligned}
$$

E-mail: f54863@sakura.kudpc.kyoto-u.ac.jp 


\begin{abstract}
This paper deals with the interference of spherical waves of thermal radiation emitted by a surface film system which consists of a metal substrate and a semi-transparent film. A spectroscopic experiment is made to reconfirm the clear interference in emission spectra of the film system. We present a theoretical model in which an electromagnetic theory for a spherical wave is combined with Planck's theory of thermal radiation. The mechanism of interference of spherical waves is discussed, and it is suggested that thermal radiation waves emitted by a number of dipoles of the metal might be coherent among each other.
\end{abstract}

Key words : thermal radiation, emission, spherical wave, interference, coherency, surface film, spectroscopic measurement 


\section{Nomenclature}

$d \quad: \quad$ (average) thickness of film, $\mathrm{m}$

$\hat{\boldsymbol{E}}(\boldsymbol{r}): \quad$ complex electric field vector at position $\boldsymbol{r}, \mathrm{V} / \mathrm{m}$

$\boldsymbol{E}_{\mathrm{O}} \quad$ : (real) electric field vector at position $\boldsymbol{r}_{\mathrm{O}}, \mathrm{V} / \mathrm{m}$

$\boldsymbol{e}_{\mathrm{q}}:$ unit vector in direction of q-polarized component of electromagnetic wave

i $\quad$ : imaginary unit

$I_{\mathrm{B}} \quad: \quad$ (spectral) intensity of blackbody radiation in vacuum, $\mathrm{W} /\left(\mathrm{m}^{3} \cdot \mathrm{sr}\right)$

$k \quad: \quad$ index of absorption, (imaginary part of $\hat{n}$ )

$k \quad$ : wavenumber of electromagnetic wave in vacuum, $\mathrm{m}^{-1}$

$\hat{\boldsymbol{k}} \quad: \quad$ complex wavenumber vector of electromagnetic wave, $\left(=\boldsymbol{k}_{\text {real }}+i \boldsymbol{k}_{\text {imag }}\right), \mathrm{m}^{-1}$

$\boldsymbol{k}_{\text {real }}, \boldsymbol{k}_{\text {imag }} \quad$ : $\quad$ real and imaginary parts of complex vector $\hat{\boldsymbol{k}}$, respectively, $\mathrm{m}^{-1}$

$n \quad: \quad$ index of refraction

$\hat{n} \quad: \quad$ optical constant, $(=n+i k)$

$R \quad: \quad$ (spectral directional-incidence specular reflection energy) reflectance

$\boldsymbol{r} \quad: \quad$ position vector of point in medium $1, \mathrm{~m}$

$\boldsymbol{r}^{\prime} \quad: \quad$ position vector of point on interface I, $\mathrm{m}$

$\hat{r} \quad: \quad$ Fresnel's complex reflection coefficient

$r_{\mathrm{O}}:$ position vector of point on hemisphere centered at point $\mathrm{O}$ in medium $1, \mathrm{~m}$

$<\boldsymbol{S}>$ : time mean of Poynting vector of electromagnetic wave, $\mathrm{W} / \mathrm{m}^{2}$

$t \quad: \quad$ time, $\mathrm{s}$

$\hat{t} \quad: \quad$ Fresnel's complex transmission coefficient

$\Delta A_{\mathrm{O}} \quad: \quad$ infinitesimal area in vicinity of position $\boldsymbol{r}_{\mathrm{O}}, \mathrm{m}^{2}$

$\Delta A_{1} \quad$ : infinitesimal area in vicinity of point $\mathrm{O},(=$ const. $), \mathrm{m}^{2}$

$\Delta A^{\prime} \quad$ : infinitesimal area in vicinity of position $\boldsymbol{r}^{\prime},(=$ const. $), \mathrm{m}^{2}$

$\Delta Q_{\mathrm{qO} l}: \quad$ energy of electromagnetic wave passing through area $\Delta A_{\mathrm{O}}, \mathrm{W}$ 
$\Delta Q_{(\mathrm{s}+\mathrm{p}) 0}:$ energy of electromagnetic wave passing through area $\Delta A^{\prime}, \mathrm{W}$

$\Delta \Omega_{0} \quad: \quad$ solid angle of observation, $\left(=\Delta \Omega_{00}\right), \mathrm{sr}$

$\Delta \Omega_{0 l} \quad: \quad$ solid angle of electromagnetic wave transmitted through interface I, sr

$\Delta \Omega_{1 l} \quad: \quad$ solid angle of electromagnetic wave incident on interface I, sr

$\varepsilon \quad: \quad$ (spectral directional) emittance

$\theta_{0}:$ emission angle of electromagnetic wave in medium 0 , (angle of observation), $\left(=\theta_{00}\right), \mathrm{rad}$

$\theta_{1}:$ emission angle of electromagnetic wave in medium $1,\left(=\theta_{10}\right), \mathrm{rad}$

$\lambda \quad$ : wavelength of electromagnetic wave in vacuum, $\mathrm{m}$

$\mu \quad: \quad$ absolute magnetic permeability of vacuum, $\mathrm{H} / \mathrm{m}$

$\sigma \quad: \quad$ rms roughness, $\mathrm{m}$

$\omega \quad: \quad$ angular frequency of electromagnetic wave, $\mathrm{rad} / \mathrm{s}$

\section{Subscripts}

B : blackbody

film : film system

$l \quad: \quad$ number of multiple reflection in film, $(l=0,1,2, \ldots)$

$\mathrm{N} \quad: \quad 15^{\circ}$-direction emission

$\mathrm{NH}: 15^{\circ}$ - incidence hemispherical reflection

NN : $15^{\circ}$ - incidence specular reflection

$\mathrm{O} \quad$ : centered at point $\mathrm{O}$

q : $\quad$ q-polarized components of electromagnetic wave, $(q=s, p)$

real, imag : real and imaginary parts of complex quantity, respectively

$\mathrm{s}, \mathrm{p} \quad$ : $\quad \mathrm{s}$ - and p-polarized components of electromagnetic wave, respectively

$(\mathrm{s}+\mathrm{p}):$ natural radiation

$0,1,2$ : medium 0 (vacuum), medium 1 (film), medium2 (substrate), respectively 
Superscripts and others

calc : calculated

$\exp : \quad$ experimental

$\wedge \quad: \quad$ complex quantity

|| $\mid$ : norm of complex quantity 


\section{Introduction}

Thermal radiation characteristics of a metal or a semi-conductor can change sensitively when a film and/or microstructure is formed on the surface. The authors [1] found that the spectra of thermal radiation emitted by a metal surface, on which a semi-transparent film is formed, show a clear phenomenon of radiation interference. The interference phenomenon in the emission spectra was impressive for us [2], but it was not a new finding. The phenomenon in such a film system had been well known empirically in the field of metallurgy [3]. However, the interference in thermally emitted radiation spectra has not been explained theoretically.

The film interference in the reflection of a plane electromagnetic wave has been well known, and has been explained enough by classical electromagnetic wave theory. On the other hand, interference of a thermally emitted radiation wave cannot be explained in a similar manner. That is, thermal radiation is spherical electromagnetic waves emitted by a number of dipoles thermally moving randomly. The spherical waves emitted by a number of neighboring dipoles have been assumed not to interfere systematically among each other. For the spherical wave to be characterized by a clear interference phenomenon, a spherical wave emitted by an individual dipole and the components of the wave multiply reflected in the film, should interfere systematically in the vicinity of the dipole. Intensity of a spherical wave attenuates in the film inversely proportional to the square of the distance from the radiation source. These conditions are different from the interference of a reflected plane wave. Kirchhoff's law which does not consider the phase of an electromagnetic wave is outside of this consideration on this interference phenomenon.

For the quantitative evaluation of the emission flux or emittance spectrum for thermal radiation, Maxwell's electromagnetic wave theory is not enough. Planck's theory of thermal radiation should be combined with the Maxwell's theory for characterizing the intensity of the emitted thermal radiation. Such a combined theoretical description has not been presented. A 
theoretical model should be presented here on the coherency of a spherical electromagnetic wave in the vicinity of radiation sources with consideration of Planck's theory.

In the thermal engineering field, thermo-photo-voltaic (TPV) conversion techniques are required, and a spectrally functional radiation emitter which emits thermal radiation in a specified wavelength band region selectively is expected to be developed [4]. The above-mentioned surface film system is promising from this engineering point of view.

In the present study, we deal with the interference of thermal radiation emitted by a film system which consists of a metal substrate and a semi-transparent film. First, a spectroscopic experiment is made on the emission and reflection of this film system. Next, a theoretical model of radiation emission is presented by combining electromagnetic theory for a spherical wave with Planck's theory of thermal radiation. The spectra calculated on the model are compared with the measured spectra to consider the mechanism of the interference of thermally emitted radiation waves. It is suggested that thermal radiation waves emitted by a number of dipoles of the metal might be coherent among each other. Also, the possibility of an effective spectrally functional radiation emitter is suggested.

\section{Procedure and Results of Spectroscopic Experiment}

\subsection{Preparation and formation of film system}

Material of the specimen of a film system is a polycrystalline nickel plate of $99.99 \%$ in chemical purity, whose size is $15 \mathrm{~mm}$ in width, $50 \mathrm{~mm}$ in length and $2 \mathrm{~mm}$ in thickness. The surface is mechanically buffed to realize the optical smoothness of the maximum roughness less than $30 \mathrm{~nm}$. The surface is heated in atmospheric air at a heating rate of $1 \mathrm{~K} / \mathrm{s}$. An oxide film is formed on the nickel surface. The surface is heated up to $1100 \mathrm{~K}$, and heating is stopped. The surface is cooled naturally to room temperature, and the surface state of $1100 \mathrm{~K}$ is frozen. 


\subsection{Spectra in formation process of film system}

In this film formation process, we measure the change of spectra of $15^{\circ}$-incidence specular reflectance $R_{\mathrm{NN}}$ and $15^{\circ}$-direction emittance $\varepsilon_{\mathrm{N}}$ of the surface simultaneously by a high-speed spectrophotometer system [1]. Figure 1 shows the results. The abscissa $\lambda$ of the figure is the wavelength of radiation in vacuum. $t$ is the time after the start of heating of the surface. At $t=600 \sim 800 \mathrm{~s}$ the surface reaches the temperature of $900 \sim 1100 \mathrm{~K}$. A surface film grows well at this stage. Corresponding to this film growth clear oscillation appears in the spectra of $R_{\mathrm{NN}}$ and $\varepsilon_{\mathrm{N}}$. With the growth of the film valleys and hills of the spectrum, oscillations appear and shift to the longer wavelength region of the spectra.

\subsection{Spectra of film system at $1100 \mathrm{~K}$}

In Figure $1, t=800 \mathrm{~s}$ corresponds to the time when heating of the surface is stopped and the surface is begun to be cooled. In the discussion on Figure 5 in Section 4, measured values of reflectance and emittance at the last stage of this heating ( $t=800 \mathrm{~s})$ are noted by $R_{\mathrm{NN}}{ }^{\exp }(1100 \mathrm{~K})$ and $\varepsilon_{\mathrm{N}}{ }^{\text {exp }}(1100 \mathrm{~K})$, respectively. The $R_{\mathrm{NN}}$ spectrum at this stage is analyzed by a spectrum surface diagnosis technique of Reference [5]. The average thickness $d$ of the formed film is $d=0.9 \mu \mathrm{m}$. The rms roughness $\sigma$ of the film surface cooled to room temperature is $\sigma=0.2 \mu \mathrm{m}$, which is measured by an optical microscope.

\subsection{Spectra of film system at $600 \mathrm{~K}$}

The surface system once cooled to room temperature is heated up to $600 \mathrm{~K}$ and kept at the temperature. Spectra of $15^{\circ}$-incidence specular reflectance $R_{\mathrm{NN}}$ and $15^{\circ}$-direction emittance $\varepsilon_{\mathrm{N}}$ are measured simultaneously by a Fourier transformation infrared spectrophotometer system [6]. This spectrophotometer was specially designed to measure the reflectance and emittance simultaneously. It takes longer time for the spectrum measurement than that by the high-speed spectrophotometer system [1], and it does not fit for the fast 
measurement of the surface in a transient process. On the other hand this FTIR system has higher sensitivity and it measures comparatively weak emission of a surface at temperatures of $600 \mathrm{~K}$ level. Also, it can measure the spectra of $R_{\mathrm{NN}}$ and emittance $\varepsilon_{\mathrm{N}}$ over the wavelength region of $\lambda=1.5 \sim 16 \mu \mathrm{m}$. Since the surface film system is known to be stable in atmospheric air at less than $700 \mathrm{~K}$, the measurement is made at $600 \mathrm{~K}$. The spectra of $R_{\mathrm{NN}}$ and $\varepsilon_{\mathrm{N}}$ measured in this experiment are noted as $R_{\mathrm{NN}}{ }^{\exp }(600 \mathrm{~K})$ and $\varepsilon_{\mathrm{N}}{ }^{\exp }(600 \mathrm{~K})$.

\subsection{Results of spectroscopic measurement}

Figure 5 as described later in Section 4.1 shows the results of the spectra of reflectance $R_{\mathrm{NN}}{ }^{\exp }(1100 \mathrm{~K})$ and $R_{\mathrm{NN}}{ }^{\exp }(600 \mathrm{~K})$ and, emittance $\varepsilon_{\mathrm{N}}{ }^{\exp }(1100 \mathrm{~K})$ and $\varepsilon_{\mathrm{N}}{ }^{\exp }(600 \mathrm{~K})$. The reflectance is that for natural radiation, and the emittance is that for emitted natural or unpolarized radiation.

\subsubsection{Experimental errors and surface scattering Measured reflectance} $R_{\mathrm{NN}}{ }^{\exp }(600 \mathrm{~K})$ is as high as 1 in the longer wavelength region of $\lambda>10 \mu \mathrm{m}$. It is considered to be caused by a background radiation error. Reflectance $R_{\mathrm{NN}}$ is low in the shorter wavelength region. It is caused mainly by the influence of the surface roughness. The surface is roughened in the process of oxide film growth. Reflected radiation is scattered over hemisphere. The reflectance $R_{\mathrm{NN}}$ is a directional reflectance for the specular reflection component. Thus, reflectance $R_{\mathrm{NN}}$ decreases particularly in the shorter wavelength region.

\subsubsection{Interference of radiation in emittance spectra In Figures 1 and 5 clear} oscillation of interference of film interference is found not only in the spectra of reflectance but in those of emittance. Interference behavior in the emitted thermal radiation $[1,2,5]$ is reconfirmed experimentally.

\subsubsection{Stability of surface state The spectra of reflectance $R_{\mathrm{NN}}{ }^{\exp }(600 \mathrm{~K})$ and} $R_{\mathrm{NN}}{ }^{\exp }(1100 \mathrm{~K})$, and the spectra of emittance of $\varepsilon_{\mathrm{N}}{ }^{\exp }(600 \mathrm{~K})$ and $\varepsilon_{\mathrm{N}}{ }^{\exp }(1100 \mathrm{~K})$ are near, respectively, to each other. Slight deviation of the wavelengths of interference in the four kinds of spectra is not due to the change in the surface state but due to the deviation of the 
wavelength correction of the two spectrophotometer systems employed. The spectrum of $\varepsilon_{\mathrm{N}}{ }^{\mathrm{exp}}(600 \mathrm{~K})$ is presumed to be near to that of $\varepsilon_{\mathrm{N}}{ }^{\mathrm{exp}}(1100 \mathrm{~K})$ even in the shorter wavelength region where the emittance could not be measured at $600 \mathrm{~K}$.

\subsubsection{Complementary relationship of reflectance and emittance In the} measured spectra of reflectance and emittance, wavelengths of hills and valleys in the spectra of emittance $\varepsilon_{\mathrm{N}}{ }^{\text {exp }}$ and those of valleys and hills in the spectra of reflectance $R_{\mathrm{NN}}{ }^{\text {exp }}$ are near to each other. With respect to the absolute values of reflectance $R_{\mathrm{N}}$ and emittance $\varepsilon_{\mathrm{N}}$, a complementary relationship of $R_{\mathrm{NN}}+\varepsilon_{\mathrm{N}}=1$ seems to hold in the longer wavelength region of $\lambda>5 \mu \mathrm{m}$ where the influence of surface scattering is weak. In this case, the directional reflectance $R_{\mathrm{NN}}$ is substantially equal to the $15^{\circ}$-incident hemispherical reflectance $R_{\mathrm{NH}}$, and the complementary relationship of $R_{\mathrm{NH}^{+}}+\varepsilon_{\mathrm{N}}=1$ holds in this wavelength region.

If we would make a simultaneous measurement of the hemispherical reflectance $R_{\mathrm{NH}}$ and emittance $\varepsilon_{\mathrm{N}}$, then we might be able to have an experimental result in which the complementary relationship of $R_{\mathrm{NH}}+\varepsilon_{\mathrm{N}}=1$ holds over the entire wavelength region including the wavelengths of strong surface scattering and interference oscillation. This relationship is formally the same as that of Kirchhoff's law. But, the law holds for the system in the thermal equilibrium and does not consider anything on the phase of the electromagnetic wave.

\section{Theoretical Modeling of Radiation Emission of Film System}

\subsection{Theory of thermal radiation emission}

Thermal radiation consists of spherical electromagnetic waves emitted by a number of dipoles. An electromagnetic wave emitted by a dipole is assumed to be incoherent to another electromagnetic wave emitted by another dipole (Figure 2). On the basis of this assumption, we present a theoretical model of an interfered electromagnetic wave of thermal radiation emitted by a film system. Behavior of an electromagnetic wave in a film system is formulated 
combining Planck's theory of thermal radiation, and we enable the description of an interfered emittance spectrum of thermal radiation of a surface film system.

\subsection{Model of radiation emission of film system}

Figure 3 explains the physical model of a film system. The film system consists of a single layer of a parallel film of thickness $d$ (medium 1) on a substrate (medium 2). The film system faces vacuum space (medium 0 ). The substrate is a strongly absorbing medium of radiation considered. The film is weakly absorbing and semi-transparent for radiation, but the self-emission is negligibly weak. The weak absorption does not affect the surface/interface reflection and transmission of the electromagnetic wave, and the reflection and transmission angles in the film can be dealt as real quantities. The interfaces I and II are assumed to be optically flat and smooth. A spherical electromagnetic wave is emitted to medium 1 by an infinitesimal area $\Delta A_{1}$ which includes a dipole at point $\mathrm{O}$ on interface II and in the medium 2 side. The spherical wave experiences $l$ times $(l=0,1,2, \ldots)$ of multiple reflection in the film of medium 1 (abbreviated in Figure 3), and is transmitted by interface I. The electric field of the $l$-th order interference wave is formed in an infinitesimal area $\Delta A^{\prime}$ ' in the vicinity of position $\boldsymbol{r}^{\prime}$ on interface I and in the medium 0 side. We observe this field at an observation point $\mathrm{P}$ far from the film system. In the calculation of emission we follow the electric field of the spherical wave which is described by complex vector quantities, and evaluate the observed energy.

\subsection{Formulation of radiation emission wave of film system}

3.3.1 s- and p-components First, we determine a coordinate system in which the origin is set at a representative point $\mathrm{O}$ of the position of a dipole, the emission source of radiation. A plane including a unit vector $\boldsymbol{N}$ on point $\mathrm{O}$ normal to interface II and a position $\boldsymbol{r}$ ' on interface $\mathrm{I}$ is named the emission plane (Figure 3 ). The spherical wave emitted at point $\mathrm{O}$ is divided into two polarization components. The component oscillating perpendicularly to 
the emission plane is named s-component, and the component oscillating parallel to the emission plane is named p-component. These two components, s-component and p-component, are represented by a subscript $\mathrm{q}(=\mathrm{s}, \mathrm{p})$ in the following. The magnitude and direction of the q-component vector of the electric field depend on $l$ of the $l$-th order wave of the multiple reflection of the emitted radiation in the film system.

\subsubsection{Electric field of spherical wave in film}

A spherical wave is emitted at

point $\mathrm{O}$ on interface II to medium 1 . The q-component $\hat{\boldsymbol{E}}_{\mathrm{q} 1}(\boldsymbol{r})$ of the complex electric field vector of the spherical wave is described at position $\boldsymbol{r}$ in medium 1, when the wave has not experienced the first reflection, by,

$$
\begin{aligned}
\hat{\boldsymbol{E}}_{\mathrm{q} 1}(\boldsymbol{r}) & =\frac{2 \pi}{\left(\boldsymbol{k}_{1, \text { real }} \cdot \boldsymbol{r}\right) \exp \left(-\boldsymbol{k}_{1, \text { imag }} \cdot \boldsymbol{r}_{\mathrm{O}}\right)} \boldsymbol{E}_{\mathrm{qO}} \exp \left(i \hat{\boldsymbol{k}}_{1} \cdot \boldsymbol{r}\right) \\
& =\frac{2 \pi}{k n_{1} r \exp \left(-k k_{1} r \mathrm{O}\right)} \boldsymbol{E}_{\mathrm{qO}} \exp \left(i k n_{1} r\right) \exp \left(-k k_{1} r\right)
\end{aligned}
$$

where,

$$
r=|\boldsymbol{r}|
$$

The complex wavenumber vector $\hat{\boldsymbol{k}}_{1}$ of the spherical wave in medium 1 is described by,

$$
\begin{aligned}
\hat{\boldsymbol{k}}_{1} & =\boldsymbol{k}_{1, \text { real }}+i \boldsymbol{k}_{1, \text { imag }} \\
& =k \hat{n}_{1} \boldsymbol{r} /|\boldsymbol{r}|
\end{aligned}
$$

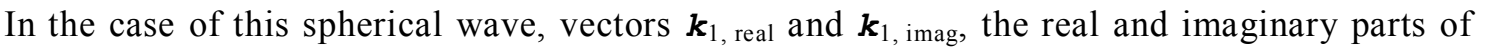
the complex vector $\hat{\boldsymbol{k}}_{1}$, are of the same direction. $\hat{n}_{1}$ is the optical constant of medium $1, k$ and $\lambda$ are wavenumber and wavelength of the electromagnetic wave in vacuum, respectively,

$$
\begin{aligned}
& \hat{n}_{1}=n_{1}+i k_{1} \\
& k=2 \pi / \lambda
\end{aligned}
$$

Electric field $\boldsymbol{E}_{\mathrm{qO}}$,

$$
\boldsymbol{E}_{\mathrm{qO}}=\hat{\boldsymbol{E}}_{\mathrm{q} 1}\left(\boldsymbol{r}_{\mathrm{O}}\right)
$$

is that at position $\boldsymbol{r}_{\mathrm{O}}$ on a hemisphere centered at point $\mathrm{O}$ and of radius,

$$
\left|\boldsymbol{r}_{\mathrm{O}}\right|=r_{\mathrm{O}}=2 \pi /\left(k n_{1}\right)=\lambda / n_{1}
$$

\subsubsection{Electric field of multiply interfered wave at film surface}


of the complex electric field vector $\hat{\boldsymbol{E}}_{\mathrm{q} 0 l}\left(\boldsymbol{r}^{\prime}\right)$ of the $l$-th wave of the spherical wave, which is emitted at point $\mathrm{O}$, has experienced $l$ times of multiple reflection in the film, and was transmitted by the interface I to the position $\boldsymbol{r}^{\prime}$ on interface $\mathrm{I}$ in the medium 0 side, is described by,

$$
\hat{\boldsymbol{E}}_{\mathrm{q} 0 l}\left(\boldsymbol{r}^{\prime}\right)=\frac{2 \pi}{\left(\boldsymbol{k}_{1, \text { real }, l} \cdot \boldsymbol{r}_{l}{ }^{\prime}\right) \exp \left(-\boldsymbol{k}_{1, \text { imag }, ~} \cdot \boldsymbol{r}_{\mathrm{O} l}\right)} \boldsymbol{E}_{\mathrm{qO} l} \exp \left(i \hat{\boldsymbol{k}}_{1 l} \cdot \boldsymbol{r}_{l}{ }^{\prime}\right) \cdot\left(\hat{r}_{\mathrm{q} 10 l}\right)^{l}\left(\hat{r}_{\mathrm{q} 12 l}\right)^{l}\left(\hat{t}_{\mathrm{q} 10 l}\right)
$$

where,

$$
\begin{aligned}
& \boldsymbol{r}_{l}{ }^{\prime}=\boldsymbol{r}^{\prime}+2 l d \boldsymbol{N} \\
& \boldsymbol{N}=(0,0,1) \\
& \hat{\boldsymbol{k}}_{1 l}=\boldsymbol{k}_{1, \text { real }, ~}+i \boldsymbol{k}_{1, \text { imag, } l} \\
& =k \hat{n}_{1} \boldsymbol{r}_{l}{ }^{\prime} /\left|\boldsymbol{r}_{l}{ }^{\prime}\right| \\
& r_{l}{ }^{\prime}=\left|\boldsymbol{r}_{l}{ }^{\prime}\right|
\end{aligned}
$$

The electric field $\boldsymbol{E}_{\mathrm{qO} l}$ in Eq. (8) characterizes the spherical wave emitted from the infinitesimal area $\Delta A_{1}$ in the vicinity of point $\mathrm{O}$ in medium 2 to medium 1 in the direction corresponding to $l$. It is described by,

$$
\boldsymbol{E}_{\mathrm{qO} l}=\left|\boldsymbol{E}_{\mathrm{qO} l}\right| \boldsymbol{e}_{\mathrm{q} l}
$$

where $\boldsymbol{e}_{\mathrm{q} l}$ is the (non-dimensional) unit vector in the direction of q-polarization component. The unit vector $\boldsymbol{e}_{\mathrm{q} l}$ is defined by the following equations.

$$
\begin{aligned}
& \boldsymbol{e}_{\mathrm{s} l}=(0,1,0) \\
& \boldsymbol{e}_{\mathrm{p} l}=\boldsymbol{e}_{\mathrm{s} l} \times \boldsymbol{r}_{l}^{\prime} /\left|\boldsymbol{e}_{\mathrm{s} l} \times \boldsymbol{r}_{l}{ }^{\prime}\right|
\end{aligned}
$$

The standard field intensity $\left|\boldsymbol{E}_{\mathrm{qO} l}\right|$ of the wave is given in Eq. (22) through discussion in Section 3.3.4. In Eq. (8) $\hat{r}_{\mathrm{qij} l}$ and $\hat{t}_{\mathrm{qij} l}$ are Fresnel's complex reflection coefficient and transmission coefficient, respectively, in the case when the q-polarization component of the $l$-th order wave propagates to interface of medium $\mathrm{i}$ and medium $\mathrm{j}$ from the medium i side. The coefficients are calculated depending on $\boldsymbol{r}_{l}$. The complex electric field vector $\hat{\boldsymbol{E}}_{\mathrm{q} 0}\left(\boldsymbol{r}^{\prime}\right)$ of the q-polarization component of superimposed/interfered wave at position $\boldsymbol{r}$ ' on interface I 
and in the medium 0 side, is described by,

$$
\hat{\boldsymbol{E}}_{\mathrm{q} 0}\left(\boldsymbol{r}^{\prime}\right)=\frac{2 \pi}{\exp \left(-\boldsymbol{k}_{1, \text { imag }} \cdot \boldsymbol{r}_{\mathrm{o}}\right)} \boldsymbol{\Sigma}_{l} \boldsymbol{E}_{\mathrm{q} \mathrm{O} l} \hat{g}_{\mathrm{q} l}
$$

where,

$$
\hat{g}_{\mathrm{q} l}=\frac{\exp \left(i \hat{\boldsymbol{k}}_{1 l} \cdot \boldsymbol{r}_{l}^{\prime}\right)}{\boldsymbol{k}_{1, \text { real }, l} \cdot \boldsymbol{r}_{l},}\left(\hat{r}_{\mathrm{q} 10 l}\right)^{l}\left(\hat{r}_{\mathrm{q} 12 l}\right)^{l}\left(\hat{t}_{\mathrm{q} 10 l}\right)
$$

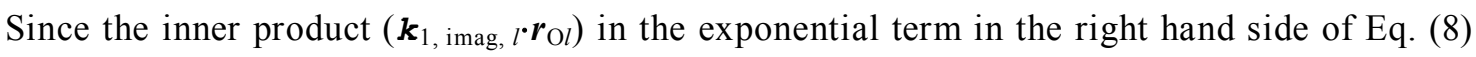
does not depend on $l$, it is written in Eq. (15) by,

$$
\boldsymbol{k}_{1, \mathrm{imag}, l} \cdot \boldsymbol{r}_{\mathrm{O} l}=\boldsymbol{k}_{1, \mathrm{imag}} \cdot \boldsymbol{r}_{\mathrm{O}}
$$

The complex electric field vector $\hat{\boldsymbol{E}}_{0}\left(\boldsymbol{r}^{\prime}\right)$ for the emission of natural radiation which consists of s- and p-polarized components equivalently is written by,

$$
\hat{\boldsymbol{E}}_{0}\left(\boldsymbol{r}^{\prime}\right)=\frac{2 \pi}{\exp \left(-\boldsymbol{k}_{1, \mathrm{~m} \text { mag }} \cdot \boldsymbol{r}_{\mathrm{o}}\right)} \Sigma_{\mathrm{q}}\left[\boldsymbol{\Sigma}_{l} \boldsymbol{E}_{\mathrm{q} O l} \hat{g}_{\mathrm{q} l}\right]
$$

3.3.4 Standard intensity of spherical wave in film We evaluate the energy $\Delta Q_{\mathrm{qO} l}$ (unit: W) of the spherical wave which passes an infinitesimal area $\Delta A_{\mathrm{O} l}$ on a hemisphere centered at position $\boldsymbol{r}_{\mathrm{O}}$ and of radius $\left|\boldsymbol{r}_{\mathrm{O}}\right|=r_{\mathrm{O}}\left(<2 d / \cos \theta_{1 l}\right)$ before the wave experiences the first reflection. The angle $\theta_{1 l}$ is the emission angle in medium 1 in the case when the wave has experienced $l$ times of multiple reflection in the film. Absorption in medium 1 is assumed to be weak enough that the direction of vector $\boldsymbol{k}_{1 \text {, real }}$ and that of vector $\boldsymbol{k}_{1 \text {, imag }}$ are the same. Thus, the angle $\theta_{1 l}$ is a real angle. The energy $\Delta Q_{\mathrm{qO} l}$ is described through the time mean $<\boldsymbol{S}_{\mathrm{q}}\left(\boldsymbol{r}_{\mathrm{O}}\right)>$ of the Poynting vector of the wave by,

$$
\begin{aligned}
\Delta Q_{\mathrm{qO} l} & =\left|<\boldsymbol{S}_{\mathrm{q} l}\left(\boldsymbol{r}_{\mathrm{O}}\right)>\right| \Delta A_{\mathrm{O} l} \\
& =k n_{1}\left|\boldsymbol{E}_{\mathrm{qO} l}\right|^{2} \Delta A_{\mathrm{O} l} /(2 \mu \omega)
\end{aligned}
$$

where $\mu$ and $\omega$ are absolute magnetic permeability of vacuum and angular frequency of the electromagnetic wave, respectively.

From another point of view, this energy $\Delta Q_{\mathrm{q} O l}$ of thermally emitted radiation should be characterized by Planck's theory of thermal radiation. The energy $\Delta Q_{\mathrm{qO} l}$ is emitted in the solid 
angle $\Delta \Omega_{\mathrm{O} l}\left(=\Delta A_{\mathrm{O} l} / r_{\mathrm{O}}^{2}\right)$ in the direction of the emission angle $\theta_{1 l}$ and attenuated by the absorption medium 1 . The energy is described by,

$$
\Delta Q_{\mathrm{qO} l}=\varepsilon_{\mathrm{q} 21 l} n_{1}^{2} \frac{I_{\mathrm{B}}}{2} \exp \left(-2 k k_{1} r_{\mathrm{O}}\right) \Delta A_{1} \cos \theta_{1 l}\left(\Delta A_{\mathrm{O} l} / r_{\mathrm{O}}{ }^{2}\right)
$$

where $I_{\mathrm{B}}$ is the intensity of blackbody radiation in vacuum. $\varepsilon_{\mathrm{q} 21 l}$ is the emittance of medium 2 to medium 1 for the q-polarized wave. It depends on temperature. At this stage, two fundamental theories are combined: electromagnetic description of a spherical wave based on the Maxwell's theory of electromagnetism, and radiative heat transfer description of diffuse blackbody radiation based on Planck's theory of thermal radiation. The emittance $\varepsilon_{\mathrm{q} 21 l}$ is calculated by using Fresnel's complex reflection coefficient by,

$$
\varepsilon_{\mathrm{q} 21 l}=1-\left|\hat{r}_{\mathrm{q} 12 l}\right|^{2}
$$

where Kirchhoff's law is assumed on the reflected and emitted intensity of radiation on the interface II. No assumption on the phase of the emitted spherical wave is adopted here.

From Eqs. (6), (19) and (20) intensity $\left|\boldsymbol{E}_{\mathrm{qO} l}\right|$ of electric field $\boldsymbol{E}_{\mathrm{qO} l}$ in Eq. (8) is written by,

$$
\begin{aligned}
\left|\boldsymbol{E}_{\mathrm{qO} l}\right|^{2} & =\frac{2 \mu \omega}{k n_{1}} \varepsilon_{\mathrm{q} 21 l} n_{1}{ }^{2} \frac{I_{\mathrm{B}}}{2} \exp \left(-2 k k_{1} r_{\mathrm{O}}\right) \Delta A_{1} \cos \theta_{1 l}\left(\frac{k n_{1}}{2 \pi}\right)^{2} \\
& =\frac{1}{(2 \pi)^{2}} 2 \mu \omega k n_{1} \varepsilon_{\mathrm{q} 21 l} n_{1}{ }^{2} \frac{I_{\mathrm{B}}}{2} \exp \left(-2 k k_{1} r_{\mathrm{O}}\right) r_{l}{ }^{2} \Delta \Omega_{1 l}
\end{aligned}
$$

The magnitude $r_{l}$ ' of vector $\boldsymbol{r}_{l}$ ' and solid angle $\Delta \Omega_{1 l}$ in this equation are described as follows,

$$
\begin{aligned}
& r_{l}{ }^{\prime}=\left|\boldsymbol{r}_{l}{ }^{\prime}\right|=(2 l+1) d / \cos \theta_{1 l} \\
& \Delta A_{1} \cos \theta_{1 l} \Delta \Omega_{\mathrm{O} l}=\Delta A^{\prime} \cos \theta_{1 l} \Delta \Omega_{1 l} \\
& \Delta \Omega_{\mathrm{O} l}=\Delta A_{\mathrm{O} l} / r_{\mathrm{O}}{ }^{2}=\Delta A^{\prime} \cos \theta_{1 l} / r_{l}{ }^{2} \\
& \Delta \Omega_{1 l}=\Delta A_{1} \cos \theta_{1 l} / r_{l}{ }^{2}
\end{aligned}
$$

3.3.5 Intensity of electric field of multiply interfered wave on film surface The magnitude of electric field $\hat{\boldsymbol{E}}_{0}\left(\boldsymbol{r}^{\prime}\right)$ in Eq. (18) is written through Eqs. (12) and (22) by,

$$
\begin{aligned}
& \left\|\hat{\boldsymbol{E}}_{0}\left(\boldsymbol{r}^{\prime}\right)\right\|^{2} \\
= & \frac{(2 \pi)^{2}}{\exp \left(-2 k k_{1} r \mathrm{o}\right)}\left\|\boldsymbol{\Sigma}_{\mathrm{q}}\left[\boldsymbol{\Sigma}_{l} \boldsymbol{E}_{\mathrm{qO} l} \hat{g}_{\mathrm{q} l}\right]\right\|^{2} \\
= & \frac{(2 \pi)^{2}}{\exp \left(-2 k k_{1} r \mathrm{o}\right)} \quad\left\|\boldsymbol{\Sigma}_{\mathrm{q}}\left[\boldsymbol{\Sigma}_{l}\left|\boldsymbol{E}_{\mathrm{q} \mathrm{O} l}\right| \boldsymbol{e}_{\mathrm{q} l} \hat{g}_{\mathrm{q} l}\right]\right\|^{2}
\end{aligned}
$$




$$
\begin{aligned}
& =\frac{(2 \pi)^{2}}{\exp \left(-2 k k_{1} r \mathrm{o}\right)} \frac{1}{(2 \pi)^{2}} 2 \mu \omega k n_{1}\left(n_{1}{ }^{2} \frac{I_{\mathrm{B}}}{2}\right) \exp \left(-2 k k_{1} r_{\mathrm{O}}\right)\left\|\Sigma_{\mathrm{q}}\left[\Sigma_{l} \varepsilon_{\mathrm{q} 21 l}{ }^{1 / 2} r_{l}, \Delta \Omega_{1 l}{ }^{1 / 2} \boldsymbol{e}_{\mathrm{q} l} \hat{g}_{\mathrm{q} l}\right]\right\|^{2} \\
& =2 \mu \omega k n_{1}\left(n_{1}{ }^{2} \frac{I_{\mathrm{B}}}{2}\right)\left\|\Sigma_{\mathrm{q}}\left[\Sigma_{l} \varepsilon_{\mathrm{q} 21 l}{ }^{1 / 2} r_{l}{ }^{\prime} \Delta \Omega_{1 l}{ }^{1 / 2} \boldsymbol{e}_{\mathrm{q} l} \hat{g}_{\mathrm{q} l}\right]\right\|^{2}
\end{aligned}
$$

\subsubsection{Conversion of solid angle accompanied by interface transmission We deal}

with the conversion of the solid angle by the interface transmission of the wave as shown in Figure 4. Solid angle $\Delta \Omega_{1 l}$ of the wave incident on interface I through an infinitesimal area $\Delta A^{\prime}$ and solid angle $\Delta \Omega_{0 l}$ after the transmission of interface I, are related to each other, by,

$$
\begin{aligned}
& \Delta A^{\prime} \cos \theta_{0 l} \Delta \Omega_{0 l}=\Delta A^{\prime} \cos \theta_{0 l} \sin \theta_{0 l} \Delta \phi \Delta \theta_{0 l} \\
& \Delta A^{\prime} \cos \theta_{1 l} \Delta \Omega_{1 l}=\Delta A^{\prime} \cos \theta_{1 l} \sin \theta_{1 l} \Delta \phi \Delta \theta_{1 l}
\end{aligned}
$$

By using Snell's equations and the differential form for transparent media [7],

$$
\begin{aligned}
& \sin \theta_{0 l}=n_{1} \sin \theta_{1 l} \\
& \Delta \theta_{0 l} \cos \theta_{0 l}=n_{1} \Delta \theta_{1 l} \cos \theta_{1 l}
\end{aligned}
$$

the following equations are obtained.

$$
\begin{aligned}
& \left(\Delta \Omega_{0 l l} \cos \theta_{0 l}\right) /\left(\Delta \Omega_{1 / l} \cos \theta_{1 l}\right)=\left\{\left(\sin \theta_{0 l}\right) /\left(\sin \theta_{1 l}\right)\right\}\left\{\left(\Delta \theta_{0 l} \cos \theta_{0 l}\right) /\left(\Delta \theta_{1 / l} \cos \theta_{1 l}\right)\right\} \\
& =n_{1}{ }^{2} \\
& \Delta \Omega_{1 l}=\left(\cos \theta_{0 l} / \cos \theta_{1 l}\right)\left(1 / n_{1}{ }^{2}\right) \Delta \Omega_{0 l} \\
& \Delta \Omega_{10}=\Delta A^{\prime} /\left(d / \cos \theta_{10}\right)^{2} \\
& \Delta \Omega_{1 l}=\Delta A^{\prime} /\left\{(2 l+1) d / \cos \theta_{1 l}\right\}^{2} \\
& =\left[\left(d / \cos \theta_{10}\right) /\left\{(2 l+1) d / \cos \theta_{1 l}\right\}\right]^{2} \Delta \Omega_{10} \\
& =\left[\cos \theta_{1 l} /\left\{(2 l+1) \cos \theta_{10}\right\}\right]^{2}\left(\cos \theta_{00} / \cos \theta_{10}\right)\left(1 / n_{1}{ }^{2}\right) \Delta \Omega_{00}
\end{aligned}
$$

We notate here symbols $\Delta \Omega_{00}, \theta_{00}$ and $\theta_{10}$ by simplified ones $\Delta \Omega_{0}, \theta_{0}$, and $\theta_{1}$, respectively. Thus, solid angle $\Delta \Omega_{1 l}$ in Eq. (27) is written by,

$$
\Delta \Omega_{1 l}=\left[\cos \theta_{1 l} /\left\{(2 l+1) \cos \theta_{1}\right\}\right]^{2}\left(\cos \theta_{0} / \cos \theta_{1}\right)\left(1 / n_{1}^{2}\right) \Delta \Omega_{0}
$$

3.3.7 Energy of radiation emitted by film system The magnitude $\left|<\boldsymbol{S}_{0}\left(\boldsymbol{r}^{\prime}\right)>\right|$ of time mean of Poynting vector of electric field $\hat{\boldsymbol{E}}_{0}\left(\boldsymbol{r}^{\prime}\right)$ in Eq. (18) is written by,

$$
\left|<\boldsymbol{S}_{0}\left(\boldsymbol{r}^{\prime}\right)>\right|=\left\|\hat{\boldsymbol{E}}_{0}\left(\boldsymbol{r}^{\prime}\right)\right\|^{2} k \cdot 1 /(2 \mu \omega)
$$

Radiation energy $\Delta Q_{0}$ which passes through the infinitesimal area $\Delta A^{\prime}$ in the vicinity of 
position $\boldsymbol{r}$ ' is written by,

$$
\begin{aligned}
\Delta Q_{0} & =\left|<\boldsymbol{S}_{0}\left(\boldsymbol{r}^{\prime}\right)>\right| \Delta A^{\prime} \cos \theta_{0} \\
& =\left\|\hat{\boldsymbol{E}}_{0}\left(\boldsymbol{r}^{\prime}\right)\right\|^{2} k \cdot 1 \cdot \Delta A^{\prime} \cos \theta_{0} /(2 \mu \omega)
\end{aligned}
$$

Accordingly, the energy $\Delta Q_{0}$ is written through Eqs. (27) and (36) by,

$$
\begin{aligned}
& \left.\Delta Q_{0}=2 \mu \omega k n_{1}\left(n_{1}{ }^{2} \frac{I_{\mathrm{B}}}{2}\right) k \cdot 1 \cdot \Delta A^{\prime}\left\{\cos \theta_{0} /(2 \mu \omega)\right\} \| \Sigma_{\mathrm{q}}\left[\Sigma_{l} \varepsilon_{\mathrm{q} 21 l}{ }^{1 / 2} r_{l}\right\rangle \Delta \Omega_{1 l}{ }^{1 / 2} \boldsymbol{e}_{\mathrm{q} l} \hat{g}_{\mathrm{q} l}\right] \|^{2} \\
& =\frac{\cos \theta_{0}}{\cos \theta_{1}} k^{2} n_{1} \frac{I_{\mathrm{B}}}{2} \Delta A^{\prime} \cos \theta_{0} \Delta \Omega_{0}\left\|\Sigma_{\mathrm{q}}\left[\quad \Sigma_{l} \quad \varepsilon_{\mathrm{q} 21 l}{ }^{1 / 2} r_{l}{ }^{\prime} \frac{\cos \theta_{1 l}}{(2 l+1) \cos \theta_{1}} \boldsymbol{e}_{\mathrm{q} l} \hat{g}_{\mathrm{q} l}\right]\right\|^{2} \\
& =\frac{\cos \theta_{0}}{\left(\cos \theta_{1}\right)^{3}} k^{2} n_{1} \frac{I_{\mathrm{B}}}{2} \Delta A^{\prime} \cos \theta_{0} \Delta \Omega_{0} \quad\left\|\Sigma_{\mathrm{q}}\left[\Sigma_{l} \quad \varepsilon_{\mathrm{q} 21 l}{ }^{1 / 2} r_{l}, \frac{\cos \theta_{1 l}}{2 l+1} \boldsymbol{e}_{\mathrm{q} l} \hat{g}_{\mathrm{q} l}\right]\right\|^{2}
\end{aligned}
$$

In this equation, only one solid angle $\Delta \Omega_{0}$, which is equal to the solid angle of observation, is included.

3.3.8 Emittance of film system The energy $\Delta Q_{0}$ is that the infinitesimal area $\Delta A^{\prime}$ on interface I of the film system emits in the solid angle $\Delta \Omega_{0}$ and in the direction of emission angle $\theta_{0}$. Let's consider the case when a blackbody of infinitesimal area $\Delta A$ ' is put at the same point $\mathrm{O}$. Radiation energy $\Delta Q_{\mathrm{B} 0}$ emitted by the blackbody in the solid angle $\Delta \Omega_{0}$ and in the emission angle $\theta_{0}$ is written by,

$$
\Delta Q_{\mathrm{B} 0}=I_{\mathrm{B}} \Delta A^{\prime} \cos \theta_{0} \Delta \Omega_{0}
$$

From Eqs. (39) and (40), (spectral directional) emittance $\varepsilon_{(\mathrm{s}+\mathrm{p}) \text {, film }}$ of the film system for natural radiation emission is written as follows,

$$
\begin{aligned}
\mathcal{E}_{(\mathrm{s}+\mathrm{p}), \text { film }} & =\Delta Q_{0} / \Delta Q_{\mathrm{B} 0} \\
& =\frac{\cos \theta_{0}}{\left(\cos \theta_{1}\right)^{3}} k^{2} n_{1}(1 / 2)\left\|\Sigma_{\mathrm{q}}\left[\quad \Sigma_{l} \varepsilon_{\mathrm{q} 21 l}^{1 / 2} r_{l}, \frac{\cos \theta_{1 l}}{2 l+1} \boldsymbol{e}_{\mathrm{q} l} \hat{g}_{\mathrm{q} l}\right]\right\|^{2}
\end{aligned}
$$

Since the vectors $\boldsymbol{e}_{\mathrm{s} l}$ and $\boldsymbol{e}_{\mathrm{p} l}$ cross orthogonally and $\left(\boldsymbol{\Sigma}_{l} \boldsymbol{e}_{\mathrm{s} l}\right)$ and $\left(\boldsymbol{\Sigma}_{l} \boldsymbol{e}_{\mathrm{p} l}\right)$ cross orthogonally, emittance $\varepsilon_{\mathrm{q}}$, film for s- and p-polarized components of radiation is written by the following equation,

$$
\varepsilon_{\mathrm{q}, \text { film }}=\frac{\cos \theta_{0}}{\left(\cos \theta_{1}\right)^{3}} k^{2} n_{1}\left\|\Sigma_{l} \varepsilon_{\mathrm{q} 21 l}{ }^{1 / 2} r_{l}, \frac{\cos \theta_{1 l}}{2 l+1} \boldsymbol{e}_{\mathrm{q} l} \hat{g}_{\mathrm{q} l}\right\|^{2}
$$

Equation (39) for the emitted radiation energy $\Delta Q_{0}$ includes $I_{\mathrm{B}}$ given by Planck's theory. On the other hand, in Eqs. (41) and (42) for emittances $\varepsilon_{(\mathrm{s}+\mathrm{p}) \text {, film }}$ and $\varepsilon_{\mathrm{q}}$, film, $I_{\mathrm{B}}$ does not 
appear. The emittance depends on temperature through the temperature dependence of the optical constant of medium 2 (substrate of the film system).

\subsection{Procedure of calculation}

In order to compare the emittance calculated by the proposed model with the measured emittance $\varepsilon_{\mathrm{N}}{ }^{\exp }(600 \mathrm{~K})$, we calculate on a film system at $T=600 \mathrm{~K}$ in which a film of $d=0.9$ $\mu \mathrm{m}$ in thickness is formed on a nickel substrate. The spectrum of optical constant $\hat{n}_{2}$ of the substrate is calculated as functions of wavelength $\lambda$ of radiation and temperature $T$, by a technique given in Reference [8]. The optical constant of the film is set at $n_{1}=2.0$ and $k_{1}=0.01$ independently of wavelength and temperature. The angle of observation of the emitted radiation is set at $\theta_{0}=15^{\circ}$. Emittances $\varepsilon_{(\mathrm{s}+\mathrm{p}) \text {, film }}, \varepsilon_{\mathrm{s}, \text { film }}$ and $\varepsilon_{\mathrm{p}}$, film in the wavelength region $\lambda=0.7 \sim 20 \mu \mathrm{m}$ are calculated. Multiple reflection in the film is calculated on $l=0,1,2, \ldots, 10$. The position vector $\boldsymbol{r}_{l}$ ' is described by,

$$
\boldsymbol{r}_{l}^{\prime}=\left(d \sin \theta_{1}, 0, d \cos \theta_{1}\right)
$$

Constants and variables in Eqs. (41) and (42) are calculated by Eqs. (4), (5), (9), (10), (11), (13), (14), (16), (21), (23) and (43), and by Snell's equations and Fresnel's equations for the reflection and refraction at interfaces I and II. In the calculation of Fresnel's complex reflection and transmission coefficients for interface $\mathrm{I}$, the optical constant of the film and the reflection and refraction angles at interface I are dealt as real quantities for considering the weak absorption of medium 1.

\subsection{Reflectance of film system}

For the comparison in Sections 4 and 5 on measured and calculated values and on reflection and emission values, we calculate the reflectance $R_{\mathrm{NN}}$ by conventional equations [9], on the case when a plane electromagnetic wave is incident on a film system of Section 3.4 and reflected specularly. 


\section{Calculated Results and Comparison with Measured Results}

\subsection{Results of spectrum calculation}

Figure 5 summarizes calculated results of spectra of reflectance $R_{\mathrm{NN}}$ and emittance $\varepsilon_{\mathrm{N}}$ of the film system with the measured results. Results of reflectance $R_{\mathrm{NN}}$ for the natural radiation, that for the s-polarized component and that for the p-polarized component are, respectively, the spectra of reflectances $R_{\mathrm{NN}(\mathrm{s}+\mathrm{p})}{ }^{\text {calc }}, \quad R_{\mathrm{NNs}}{ }^{\text {calc }}$ and $R_{\mathrm{NNp}}{ }^{\text {calc }}$ in the figure. Results of emittance $\varepsilon_{\mathrm{N}}$ for the natural radiation, that for the s-polarized component and that for the p-polarized component are, respectively, the spectra of emittances $\varepsilon_{\mathrm{N}(\mathrm{s}+\mathrm{p})}{ }^{\mathrm{calc}}\left(=\varepsilon_{(\mathrm{s}+\mathrm{p}), \text { film }),} \varepsilon_{\mathrm{Ns}}{ }^{\text {calc }}\left(=\varepsilon_{\mathrm{s}}\right.\right.$, film $)$ and $\varepsilon_{\mathrm{Np}}{ }^{\text {calc }}\left(=\varepsilon_{\mathrm{p}}\right.$, film $)$ in the figure. Difference of $R_{\mathrm{NN}(\mathrm{s}+\mathrm{p})}{ }^{\text {calc }}, R_{\mathrm{NNs}}{ }^{\text {calc }}$ and $R_{\mathrm{NNp}}{ }^{\text {calc }}$ and that of $\varepsilon_{\mathrm{N}(\mathrm{s}+\mathrm{p})}{ }^{\text {calc }}, \varepsilon_{\mathrm{Ns}}{ }^{\text {calc }}$ and $\varepsilon_{\mathrm{Np}}{ }^{\text {calc }}$ are small. Figure 5 also shows the spectra of reflectance $R_{\mathrm{NN}}{ }^{\text {calc }}$ (specular) and emittance $\varepsilon_{\mathrm{N}}{ }^{\text {calc }}$ (specular) of the bare optically smooth nickel surface for comparison.

\subsection{Calculated values of reflectance and emittance}

The calculated reflectance is for the plane wave, and the emittance is for the spherical wave. In spite of this difference, wavelengths of the valleys and hills of the oscillation in the calculated spectra of reflectance $R_{\mathrm{NN}}{ }^{\text {calc }}$, and those of hills and valleys of the oscillation in the calculated spectra of emittance $\varepsilon_{\mathrm{N}}{ }^{\text {calc }}$ are, respectively, near to each other. In this calculation on a flat and smooth film system the directional reflectance $R_{\mathrm{NN}}$ is equal to the hemispherical reflectance $R_{\mathrm{NH}}$. If we assume that Kirchhoff's law for thermal equilibrium systems is valid, then the following complementary relationship,

$$
R_{\mathrm{NN}}{ }^{\mathrm{calc}}+\varepsilon_{\mathrm{N}}{ }^{\mathrm{calc}}=R_{\mathrm{NH}}{ }^{\mathrm{calc}}+\varepsilon_{\mathrm{N}}{ }^{\mathrm{calc}}=1
$$

holds between reflectance $R_{\mathrm{NN}}{ }^{\text {calc }}$ and emittance $\varepsilon_{\mathrm{N}}{ }^{\text {calc }}$. This quantitative relationship seems to hold except for the calculated values of $R_{\mathrm{NN}}{ }^{\text {calc }}$ and $\varepsilon_{\mathrm{N}}{ }^{\text {calc }}$ in the wavelength regions of interference hills in the $\varepsilon_{\mathrm{N}}{ }^{\text {calc }}$ spectrum. In the wavelength regions of interference hills in the 
$\varepsilon_{\mathrm{N}}{ }^{\text {calc }}$ spectrum, the height of the hills in the emittance spectrum is smaller than the depth of the valleys in the $R_{\mathrm{NN}}{ }^{\text {calc }}$ spectrum.

\subsection{Calculated and measured values of reflectance and emittance}

We compare the calculated and measured spectra of reflectance and emittance. Since the influence of surface roughness of the film system is neglected in the calculation, values of reflectance $R_{\mathrm{NN}}{ }^{\text {calc }}$ are calculated higher than the measured values of $R_{\mathrm{NN}}{ }^{\text {exp }}$ in the shorter wavelength region where the influence of surface scattering on the directional reflectance $R_{\mathrm{NN}}{ }^{\text {exp }}$ is stronger. Since the emittance increase by surface roughness in the surface system of the experiment is not considered in the calculation, values of emittance $\varepsilon_{\mathrm{N}}{ }^{\text {calc }}$ are lower than those of $\varepsilon_{\mathrm{N}}{ }^{\text {exp }}$ particularly in the shorter wavelength region. Also, since self-emission of the film is neglected in the calculation, the values of the calculated emittance $\varepsilon_{\mathrm{N}}{ }^{\text {calc }}$ are lower. But, with respect to the radiation interference, the wavelengths of interference in the calculated spectra of $R_{\mathrm{NN}}{ }^{\text {calc }}$ and $\varepsilon_{\mathrm{N}}{ }^{\text {calc }}$ agree well with those in the measured spectra of $R_{\mathrm{NN}}{ }^{\text {exp }}$ and $\varepsilon_{\mathrm{N}}{ }^{\text {exp }}$. The hills of interference in the calculated spectrum of $\varepsilon_{\mathrm{N}}{ }^{\text {calc }}$ are less sharp than the hills of interference in the measured spectrum of $\varepsilon_{\mathrm{N}}{ }^{\exp }$.

\section{Interference of Emitted Thermal Radiation}

\subsection{Wavelength of interference and phase of spherical wave}

Interference in an emittance spectrum for a spherical wave is different from that in a reflectance spectrum for a plane wave. The interference occurs as a result of multiple reflection in the film system. The spherical wave propagates over various directions. This fact may imply that the wavelengths of interference depend on the direction of observation, and that the emittance spectrum lacks the sharpness in the spectrum oscillation. But, it is not the case in the measured results in Figure 5. It is explained by the following facts. The refractive 
index of the film is of an order of $n_{1}=2$, and the radiation in the direction of emission angle of $15^{\circ}$, the angle of observation in the present study, is the interfered components of spherical waves emitted by a dipole on interface II of the film system and propagates in the direction of $\theta_{l}<7.4^{\circ}$ in the film. That is, direction, phase and wavelength of the interference of the observed emission wave are not so much different from those in the case of the reflection of the plane wave.

\subsection{Sharpness and amplitude of spectrum oscillation}

The amplitude of interference oscillations in the calculated emittance spectrum is not so sharp as that in the calculated reflectance spectrum. The following should be noticed. The interference of the wave in the film system occurs mainly among the 0 th-order wave $(l=0)$ and the 1 st-order wave $(l=1)$. In a weakly absorbing medium, intensity of a spherical wave is attenuated inversely proportional to the square of the distance from the radiation source. The intensity of the 1 st-order wave decreases in the film to be $1 / 9$ of that of the 0 th-order wave. Equivalent interference of the 1st- and 0th-order waves, as is the case in the film reflection of plane waves, can not be realized in the case of emitted spherical waves. Therefore interference in the calculated emittance spectrum is far less clear than that in the calculated reflectance spectrum.

\subsection{Interference of radiation emitted by a number of dipoles}

The present theoretical model describes the interference of the emitted thermal radiation qualitatively well. But, the spectrum oscillation in the calculated emittance spectrum is less sharp than that in the measured emittance spectrum, and the values of the calculated emittance are lower than those of the measured emittance. Since the roughness of the surface of the experiment influences the measured spectrum to round the interference effect in the oscillating spectra and to increase the values of emittance inversely, it cannot be the origin of the deviation of the calculated and measured emission spectra. With respect to the sharpness 
of the spectrum oscillation, the measured emittance spectrum is rather near to the measured reflectance spectrum. This point is important in considering the validity of the model proposed in Section 3. Considering the discussion in Sections 4.3 and 5.2, the deviation in calculated and measured emission spectra may be caused by the assumption in Section 3.1 on the incoherency of the emitted radiation wave by a number of dipoles in the metal substrate.

In the present film system the radiation sources of the dipole emission are conduction electrons of a metal. Thermal movement of a number of conduction electrons might not be independent among each other, but the electromagnetic fields of the emitting dipoles might interact with each other. Electromagnetic waves of thermal radiation emitted by a number of dipoles in the metal might be coherent among each other. If it is the case, the electromagnetic field of multiple reflection of the spherical waves in the film system which emits thermal radiation may be similar to the field of multiple reflection of the plane waves of the reflection. Theoretical calculation on such a model of coherent dipole emission sources would estimate that the interference of the waves of $l=0$ and $l=1,2,3, \ldots$ can be similar to that in the case of reflection of a plane wave, and the interference in the emittance spectrum as sharp as that in the measured spectrum.

\subsection{Spectrally functional emitter of thermal radiation}

In the measured emittance spectra in Figure 5, emittance is high in the longer interference hill wavelength region. The present film system emits the radiation of the wavelength band region selectively. On the other hand, radiation energy in the shorter wavelength side less than $\lambda=2 \mu \mathrm{m}$ is weak for the sake of Planck's distribution at the temperature of a $600 \mathrm{~K}$ level irrespective of the higher emittance values in the shorter wavelength region. That is, the spectral function of the present film system is excellent. The wavelength of interference of the film system is easily controlled in the film formation process as shown in Figure 1. The film system can be produced easily over a wide surface area of an order of $\mathrm{m}^{2}$. The present film system is prospective for a radiation emitter for the 
new thermal energy engineering.

\section{Concluding Remarks}

In the present study, a spectroscopic experiment and a model calculation of electromagnetism have been made on a spherical wave of thermal radiation emitted by a film system which consists of a metal substrate and a semi-transparent film. The following conclusions have been made:

(1) It has been reconfirmed experimentally that thermal radiation emitted by this film system is characterized by a clear interference phenomenon.

(2) A theoretical model has been presented to describe the interference in thermal radiation emission of this film system. In the model an electromagnetic theory for a spherical wave is combined with Planck's theory of thermal radiation.

(3) It has been suggested that thermal radiation waves emitted by a number of dipoles of a metal might be coherent among each other.

(4) This film system is prospective as a spectrally functional emitter of radiation which emits radiation in a specified wavelength band region selectively. 


\section{Acknowledgments}

This work was partially supported by Grant-in-Aids for Scientific Research ((A)17206019) and Grant-in-Aid for Young Scientists ((B) 17760165) of The Ministry of Education, Culture, Sports, Science and Technology, Japan. 


\section{References}

[1] Wakabayashi, H. and Makino, T., A New Spectrophotometer System for Measuring Thermal Radiation Phenomena in a 0.30-11 $\mu \mathrm{m}$ Wavelength Region, Measurement Science and Technology, vol. 12, no. 12 (2001), pp. 2113-2120.

[2] Makino, T., Thermal Radiation Spectroscopy for Heat Transfer Science and for Engineering Surface Diagnosis, "Heat Transfer 2002", vol.1, (2002), pp. 55-66, Taine, J. ed., Elsevier, Paris.

[3] Iuchi, T. and Furukawa, T., Emissivity- Compensated Radiation Thermometry, Proceedings of IMEKO 2000 International Measurement Confederation 16th IMEKO World Congress, vol. 6, (2000), pp. 365-369.

[4] Hanamura, K. and Kumano, T., Thermophotovoltaic Power Generation by Super-Adiabatic Combustion in Porous Quartz, Thermal Science and Engineering, vol. 10, no. 4 (2002), pp. 9-10.

[5] Makino, T. and Wakabayashi, H., Thermal Radiation Spectroscopy Diagnosis for Temperature and Microstructure of Surfaces, JSME International Journal, ser.B, vol. 46, no. 11 (2003), pp.500-509.

[6] Makino, T., Wakabayashi, H. and Matsumoto, M., Interference of Spherical Wave of Thermal Radiation Emitted by a Film System and a Grating System, Proceedings of the First International Forum on Heat Transfer, (2004), pp.185-186.

[7] Brewster, M. Q., "Thermal Radiative Transfer and Properties", (1992), pp. 114-156, John Wiley \& Sons, New York.

[8] Makino, T., Wakabayashi, H. and Matsumoto, M., Interference of Spherical Wave of Thermal Radiation Emitted by a Film System and a Grating System, Proceedings of the First International Forum on Heat Transfer, (2004), pp.185-186.

[9] Heaven, O. S., “Optical Properties of Thin Solid Films”, (1965), pp. 46-95, Dover Pub. 
Inc., New York. 


\section{Figure Captions}

Figure 1 Spectrum transition of reflectance $R_{\mathrm{NN}}$ and emittance $\varepsilon_{\mathrm{N}}$ of a nickel surface in a film formation process

Figure 2 Interference of thermal radiation emitted by a film system

Figure 3 Physical model of a film system

Figure 4 Conversion of solid angles for the $l$-th spherical wave

Figure 5 Spectra of reflectance $R_{\mathrm{NN}}$ and emittance $\varepsilon_{\mathrm{N}}$ of the film system (measured and calculated) 


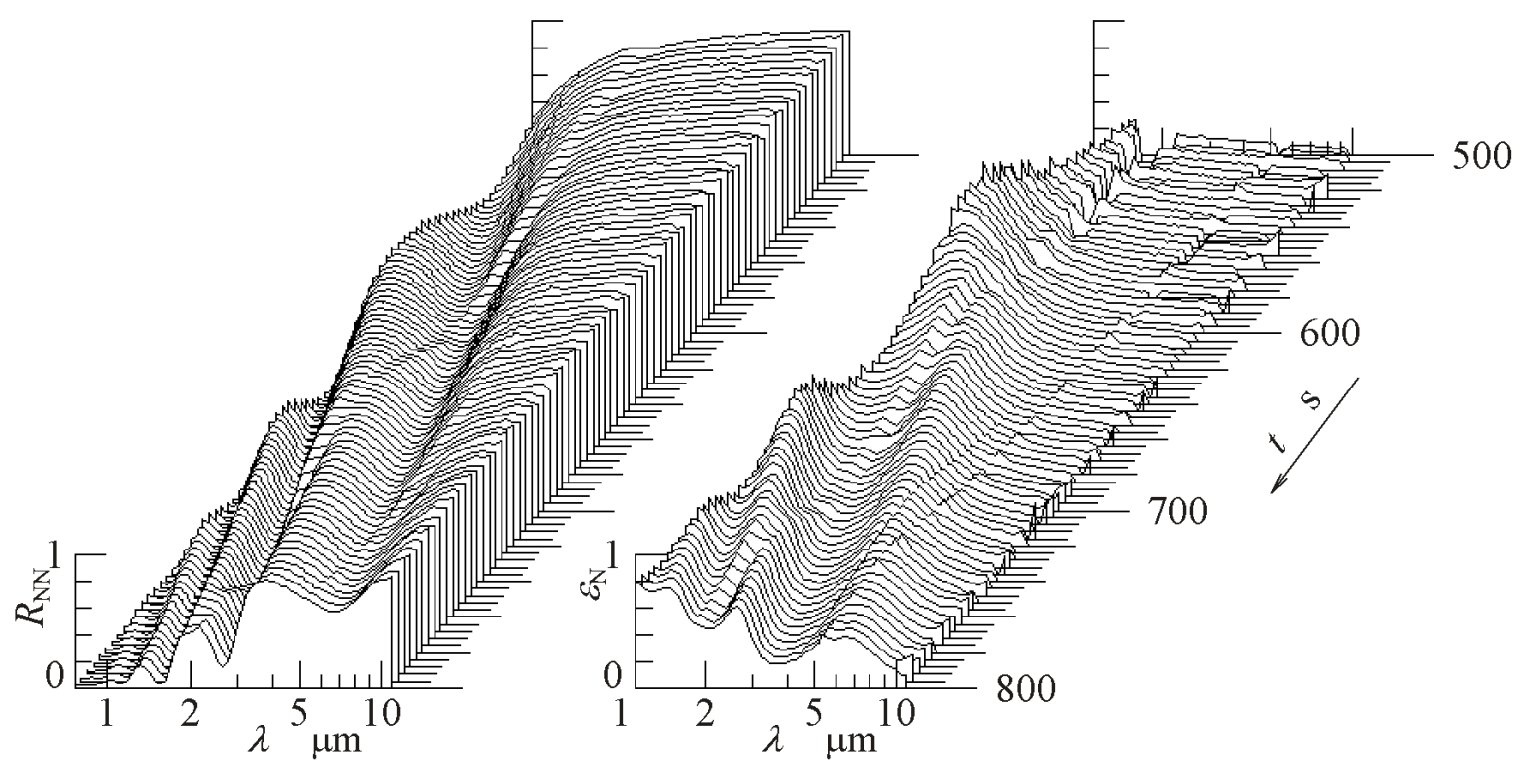

Figure 1 Spectrum transition of reflectance $R_{\mathrm{NN}}$ and emittance $\varepsilon_{\mathrm{N}}$ of a nickel surface in a film formation process 


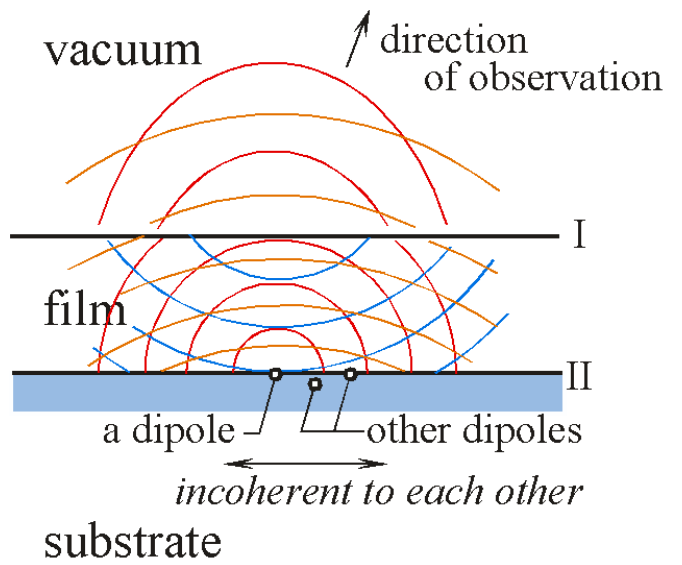

Figure 2 Interference of thermal radiation emitted by a film system 


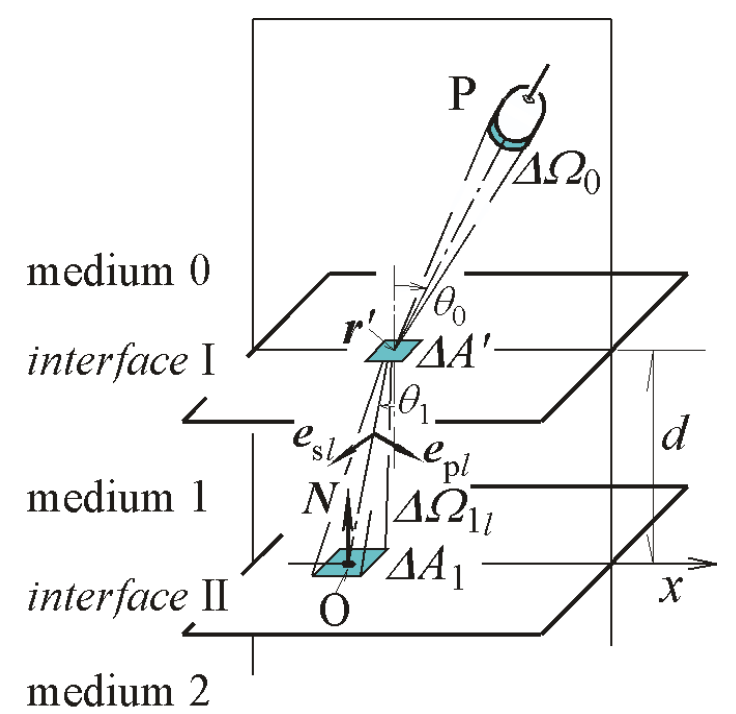

Figure 3 Physical model of a film system 


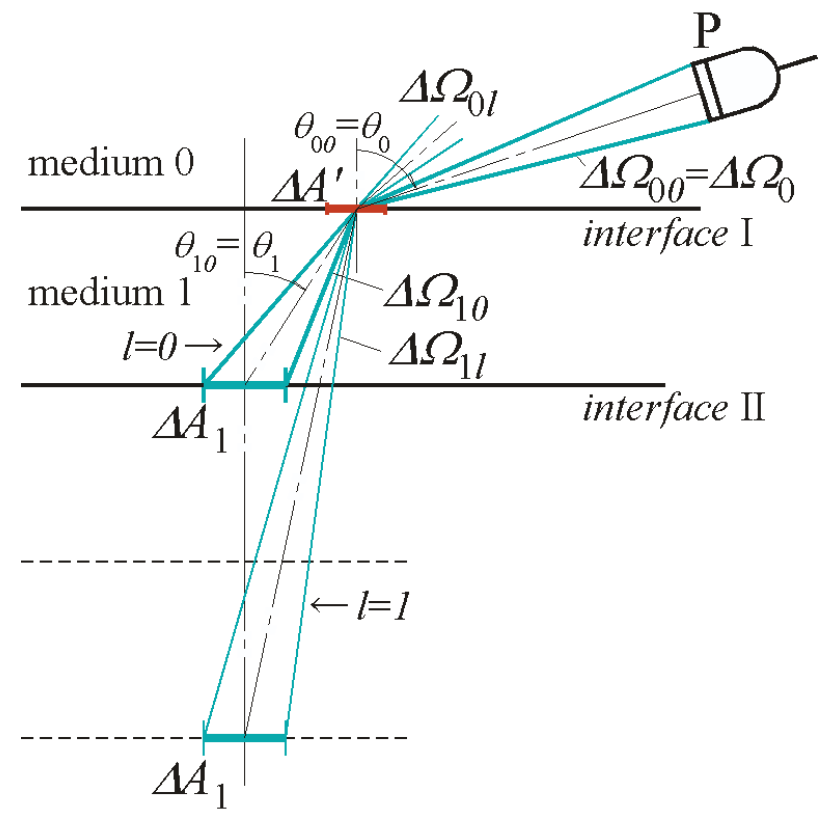

Figure 4 Conversion of solid angles for the $l$-th spherical wave 


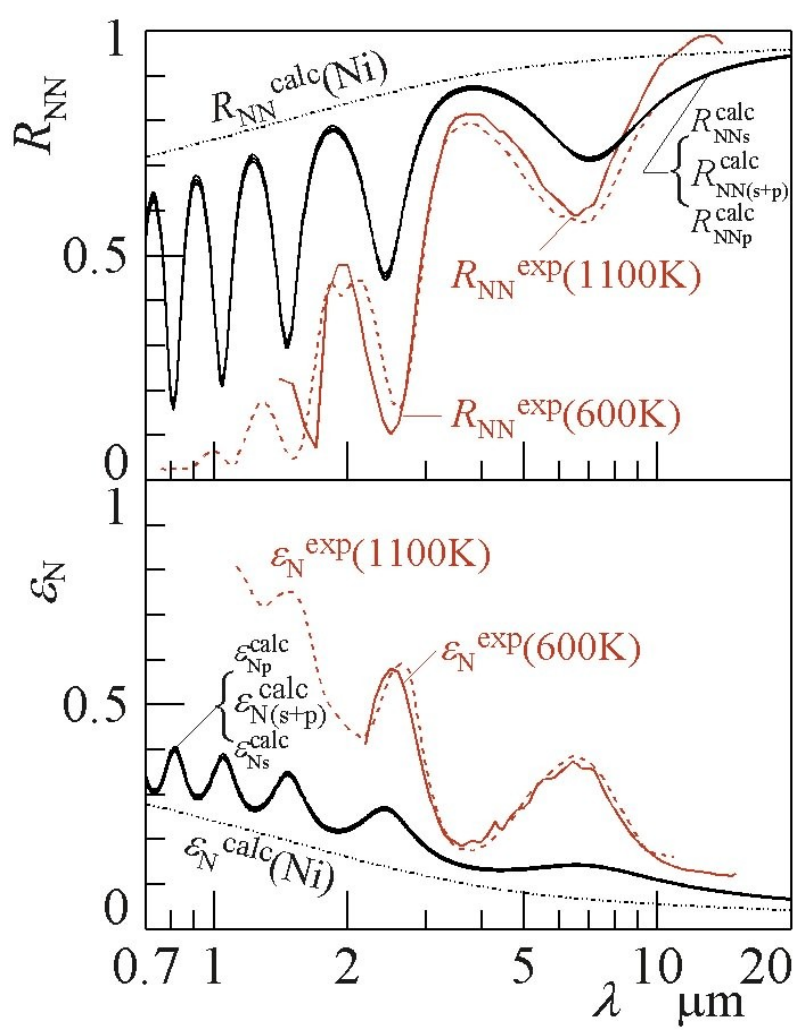

Figure 5 Spectra of reflectance $R_{\mathrm{NN}}$ and emittance $\varepsilon_{\mathrm{N}}$ of the film system (measured and calculated) 